Pacific Journal of 


\title{
DETERMINING KNOT TYPES FROM DIAGRAMS OF KNOTS
}

\author{
JOHN R. MARTIN
}

\begin{abstract}
The word of knot and the characteristics of its double points, both of which may be read from the diagram of knot, are used to give necessary and sufficient conditions for two (oriented) knots to belong to the same (oriented) knot type.
\end{abstract}

1. Introduction. A knot is a circle imbedded as a polygon in 3-dimensional space $\boldsymbol{R}^{3}$. Two knots $K$ and $L$ are called equivalent if there is an autohomeomorphism $h$ of $\boldsymbol{R}^{3}$ such that $h(K)=L$. Each equivalence class is called a knot type. For oriented knots a stronger equivalence may be defined: Two oriented knots $K$ and $L$ are called O-equivalent if there is an orientation preserving autohomeomorphism $h$ of $R^{3}$ such that $h$ maps $K$ onto $L$ so their orientations match. In this case each equivalence class is called an oriented knot type.

In [8] D. E. Penney uses the diagram of a knot to define a "word" for the knot and obtain sufficient conditions for two knots to belong to the same knot type. Penney's results have been generalized by L. B. Treybig in [12] where the concept of the "boundary collection" of a knot is used to give necessary conditions for two knots to belong to the same knot type. The purpose of this paper is to use the word of a knot and the characteristics of its double points, both of which may be read from the diagram of a knot, to give necessary and sufficient conditions for two (oriented) knots to belong to the same (oriented) knot type.

The preliminaries needed for our main results are given in $\S 2$. In $\S 3$ a relationship between the word of a knot and the characteristics of its double points is derived. This relationship is used to obtain Theorem 3.4 which yields sufficient conditions for two oriented knots to belong to the same oriented knot type. In $\S 4$ the following two principal results are obtained: Theorem 4.3 states that two prime knots $K$ and $L$ are equivalent iff there exists a certain finite sequence of words relating a word of $K$ to a word of $L$. Theorem 4.4 states that two oriented knots $K$ and $L$ are $O$-equivalent iff there exists a certain finite sequence of words and characteristics relating the word and characteristic of $K$ to those of $L$.

We remark that in each of Theorems 4.3 and 4.4 the sufficiency part follows from the results developed in $\S 3$ while the necessity is an immediate consequence of the classical work of Alexander and Briggs [1].

In $\S 5$ the group of a prime word is defined and this is used in 
Theorem 5.1 to give necessary and sufficient conditions for a group to be a knot group.

2. Preliminaries and basic lemma. Let $K$ be an oriented knot in regular position with respect to the parallel projection $p$ from $\boldsymbol{R}^{3}$ onto the $x y$-plane $R^{2}$. Suppose $K$ has $n$ double points $d_{1}, \cdots, d_{n}$ and the corresponding overcrossing (undercrossing) points are denoted by $o_{1}, \cdots, o_{n}\left(u_{1}, \cdots, u_{n}\right)$. With each $o_{i}\left(u_{i}\right)$ we associate the syllable $d_{i}\left(d_{i}^{-1}\right)$. The oriented knot $K$ determines a cyclically ordered sequence of $2 n$ crossing points. By arbitrarily designating one crossing point as the first and replacing each point in the resulting sequence by its associated syllable we obtain a word for $K$ (Fig. 1). Words $x_{1}^{a_{1}} \cdots x_{2 m}^{a_{2 m}}$ and $y_{1}^{b_{1}} \cdots y_{2 n}^{b_{2 n}}$ are defined to be equivalent if $m=n$ and there is a cyclic permutation $\alpha$ of $1, \cdots, 2 m$ such that $x_{i}=x_{j}$ iff $y_{\alpha(i)}=y_{\alpha(j)}$ and $a_{i}=b_{\alpha(j)}, a_{j}=b_{\alpha(j)}$. A nonempty word of a knot is called prime if it contains no proper segment $S$ such that $s \in S$ iff $s^{-1} \in S$. (For instance, $a b^{-1} c a^{-1} b c^{-1}$ is prime but $x a b^{-1} c a^{-1} b c^{-1} x^{-1}$ is not.)

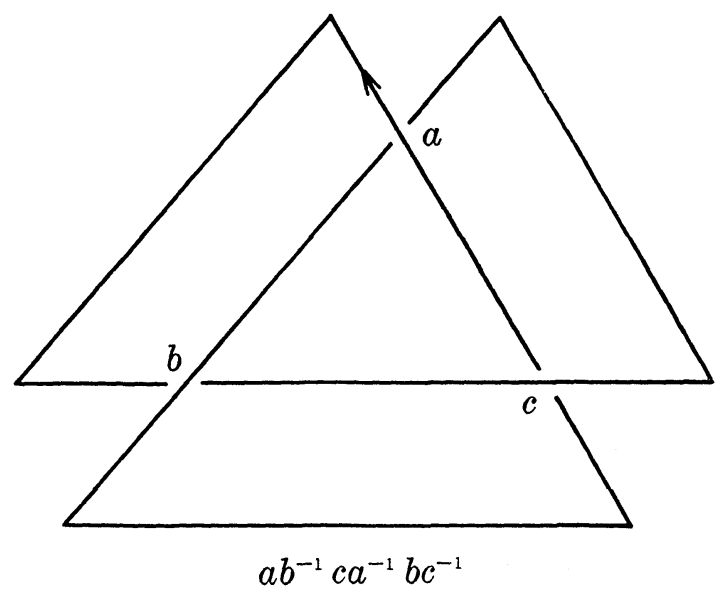

FIGURE 1

We remark that the results of [6] or [10] can be used to give necessary and sufficient conditions for a given word to be the word associated with the diagram of an oriented knot.

To each double point $d$ of a diagram of an oriented knot we

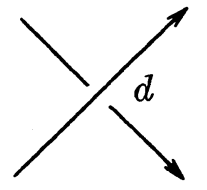

$\varepsilon(d)=1$

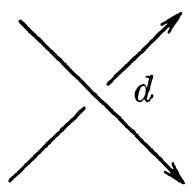

$\varepsilon(d)=-1$ 
associate an integer $\varepsilon(d)= \pm 1$, called the characteristic of $d$ (see p. 18 of [9]), according as the directed underpass does or does not cross under the directed overpass from left to right so as to preserve a left-handed screw. We note that $\varepsilon$ is independent of the orientation assigned to the knot (Fig. 2).

We shall call the directed arc between two successive crossing points of an oriented knot $K$ a boundary arc. Thus to each pair of consecutive syllables of a word for $K$ there corresponds a unique boundary arc on $K$. We now state a lemma which follows from the proof of Theorem 2 of [8].

Basic Lemma 2.1. Let $K$ and $L$ be two oriented knots possessing equivalent prime words. Then there is an isotopic deformation of $\boldsymbol{R}^{3}$, fixed on a neighborhood of double points of $K$, which transforms $K$ into a knot $K^{\prime}$ such that

(a) The oriented projections $p\left(K^{\prime}\right)$ and $p(L)$ are plane equivalent under an autohomeomorphism of $\boldsymbol{R}^{2}$ which maps projected boundary arcs of $K^{\prime}$ onto the corresponding projected boundary arcs of $L$.

(b) $K$ and $L$ belong to the same knot type.

3. The prime factorization of a word. If $W$ is a nonempty word which is not prime, then it can be written in the form $A B C$ where $A C, B$ are nonempty words such that $b \in B$ iff $b^{-1} \in B$. Clearly $A C$ and $B$ themselves are words for some knot. Moreover, $W$ can be written in the above form where $B$ is a prime word. If $A C$ is not prime the process may be repeated and so on. In this manner every nonempty word $W$ may be factored into a finite number of prime words, called the prime factors of $W$. Furthermore, it is easy to see that this factorization is unique except possibly for the order of the prime factors.

Definition 3.1. Let $P(Q)$ denote the set of prime factors of the nonempty word $U(V)$. Then $U$ and $V$ have equivalent prime factorizations iff there is a bijection $h$ between $P$ and $Q$ such that $W \in P$ implies $h(W)$ is equivalent to $W$.

Lemma 3.2. Let $K\left(K^{\prime}\right)$ be a knot with double points $d_{1}, \cdots$, $d_{n}\left(d_{1}^{\prime}, \cdots, d_{n}^{\prime}\right)$ and prime word $W\left(W^{\prime}\right)$. Suppose $W$ is equivalent to $W^{\prime}$ and $d_{i}$ corresponds to $d_{i}^{\prime}(i=1, \cdots, n)$ under this equivalence. Then $\varepsilon\left(d_{1}\right)=\varepsilon\left(d_{1}^{\prime}\right)$ implies $\varepsilon\left(d_{2}\right)=\varepsilon\left(d_{i}^{\prime}\right)$ for $i=2, \cdots, n$.

Proof. By Lemma 2.1 we may assume that there is an autohomeomorphism $h$ of $\boldsymbol{R}^{2}$ mapping the oriented projection $p(K)$ onto the oriented projection $p\left(K^{\prime}\right)$ so that the projected boundary arcs of 
$K$ are mapped onto the corresponding projected boundary arcs of $K^{\prime}$. It is well known (p. 158 of [3]) that we may color the unbounded region of $p(K)\left(p\left(K^{\prime}\right)\right)$ white and then alternately color the bounded regions of $p(K)\left(p\left(K^{\prime}\right)\right)$ black and white so that every projected boundary arc of $K\left(K^{\prime}\right)$ lies on the boundary of precisely one black region and one white region. We may suppose that the double points of $K$ have been labelled so that there is a projected boundary arc $d_{1} a d_{2}$ joining $d_{1}$ to $d_{2}$. The arc $d_{1} a d_{2}$ lies on the boundary of precisely one black region, say $D$. Since $K$ has a prime word it follows from Theorem 9 of [10] that $\mathrm{Bd} D$ is a simple closed curve, and by Theorem 7 of [10] the union of a finite number of projected boundary arcs form an arc $d_{2} b d_{1}$ such that $\operatorname{Bd} D=d_{1} a d_{2} \cup d_{2} b d_{1}$.

Since $h$ preserves projected overpasses (underpasses) and their orientations, $\varepsilon\left(d_{1}\right)=\varepsilon\left(d_{1}^{\prime}\right)$ implies that the projected overpass at $d_{1}$ can be rotated through black regions by a clockwise rotation onto the projected underpass at $d_{1}$ iff the projected overpass at $d_{1}^{\prime}$ can be rotated through black regions by a clockwise rotation onto the projected underpass at $d_{1}^{\prime}$. It then follows the preceding statement is true if $d_{1}\left(d_{1}^{\prime}\right)$ is replaced by $d_{2}\left(d_{2}^{\prime}\right)$, and therefore $\varepsilon\left(d_{2}\right)=\varepsilon\left(d_{2}^{\prime}\right)$. By successively applying this procedure $n-1$ times we obtain $\varepsilon\left(d_{i}\right)=$ $\varepsilon\left(d_{i}^{\prime}\right)$ for $i=2, \cdots, n$.

Since the mirror image of a knot $K$ can be rotated to yield a knot $L$ such that $K$ and $L$ have equivalent words and the characteristics of corresponding double points have opposite signs, we obtain

Corollary 3.3. Let $K\left(K^{\prime}\right)$ be a knot with double points $d_{1}, \cdots$, $d_{n}\left(d_{1}^{\prime}, \cdots, d_{n}^{\prime}\right)$ and prime word $W\left(W^{\prime}\right)$. Suppose $W$ is equivalent to $W^{\prime}$ and $d_{i}$ corresponds to $d_{i}^{\prime}(i=1, \cdots, n)$ under this equivalence. Then either $\varepsilon\left(d_{i}^{\prime}\right)=\varepsilon\left(d_{i}\right)(i=1, \cdots, n)$ or $\varepsilon\left(d_{i}^{\prime}\right)=-\varepsilon\left(d_{i}\right)(i=1, \cdots, n)$.

THEOREM 3.4. Let $K(L)$ be an oriented knot with word $U(V)$ whose prime factors are $U_{1}, \cdots, U_{m}\left(V_{1}, \cdots, V_{m}\right)$. Suppose $U$ and $V$ have equivalent prime factorizations with $U_{i}$ being equivalent to $V_{i}(i=$, $\cdots, m)$. Then, if the characteristic of a double point of $U_{i}$ is equal to the characteristic of the corresponding double point of $V_{i}(i=1, \cdots, m)$, the oriented knots $K$ and $L$ belong to the same oriented knot type.

Proof. Now $K(L)$ can be represented as a composition of oriented knots $K_{1}, \cdots, K_{m}\left(L_{1}, \cdots, L_{m}\right)$ whose words are $U_{1}, \cdots, U_{m}\left(V_{1}, \cdots, V_{m}\right)$. Since the set of oriented knot types form a commutative semigroup (indeed, with unique factorization) [p. 140,3], it suffices to prove the theorem in the case when $U=U_{1}$ and $V=V_{1}$.

By Lemma 2.1 we may assume that there is an autohomeomorphism $h$ of $R^{2}$ mapping the oriented projection $p(K)$ onto the oriented pro- 
jection $p(L)$ so that the projected boundary arcs of $K$ are mapped onto the corresponding boundary arcs of $L$. Let $R_{1}\left(R_{2}\right)$ denote the unbounded region of $K(L)$. Without loss of generality we may assume that $\mathrm{Bd} R_{i}(i=1,2)$ lies on a circle $C_{i}$ except in a neighborhood of double points. By Lemma 3.2 the characteristics of corresponding double points are equal. It follows that if $d_{i} d_{k}$ is a projected boundary arc of $K$, then $d_{2} d_{k}$ induces a clockwise orientation on $C_{1}$ iff $h\left(d_{i} d_{k}\right)$ induces a clockwise orientation on $C_{2}$. Hence we may suppose that $h$ is the identity on $\boldsymbol{R}^{2} \backslash \operatorname{Int} C$ where $C$ denotes a circle in $\boldsymbol{R}^{2}$ whose interior contains $p(K)$ and $p(L)$. Let $f(x, y, z)=(h,(x, y), z)$. Then $f$ is an orientation preserving autohomeomorphism of $\boldsymbol{R}^{3}$. Since $f(K)$ and $L$ obviously belong to the same oriented knot type the result follows.

If in Theorem 3.4 it is only assumed that $U$ and $V$ have equivalent prime factorizations, then it follows from (b) of Lemma 2.1 that $K(L)$ can be represented as a composition of knots $K_{1}, \cdots, K_{m}\left(L_{1}, \cdots, L_{m}\right)$ where $K_{i}$ and $L_{i}$ belong to the same knot type $(i=1, \cdots, m)$. Consequently we have

THEOREM 3.5. Let $K$ and $L$ be two knots which may be given orientations so that their words have equivalent prime factorizations. If either $K$ or $L$ is a prime knot, then they belong to the same knot type.

We note that it is possible for inequivalent nonprime knots to have words possessing equivalent prime factorizations. For instance, it is well known that the square and granny knots belong to different knot types ([2]). However, diagrams can be chosen for these two knots so as to yield identical words.

4. Diagram transformations. We remark that due to the work of Graeub ([5]) and Moise ([7]) the classical notion of equivalence for (oriented) knots (see [1]) agrees with the definitions given in $\S 1$. In [1] Alexander and Briggs have shown that two oriented knots belong to the same oriented knot type iff the diagram of one can be transformed into the diagram of the other by a finite sequence of the following types of deformations or their inverses:

( I ) A boundary arc acquires a loop which creates a new double
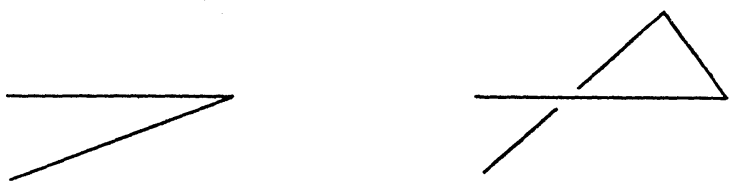

Figure 3 
point (Fig. 3).

(II) One boundary arc passes under another with the creation of two new double points (Fig. 4).
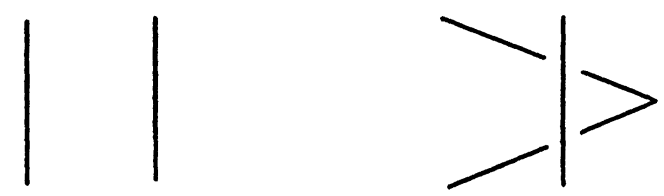

FIGURE 4

(III) If there is a region bounded by three double points and three projected boundary arcs where the endpoints of one of the boundary arcs are undercrossing points, then any one of the boundary arcs may be deformed past the crossing points determined by the other two boundary arcs (Fig. 5).
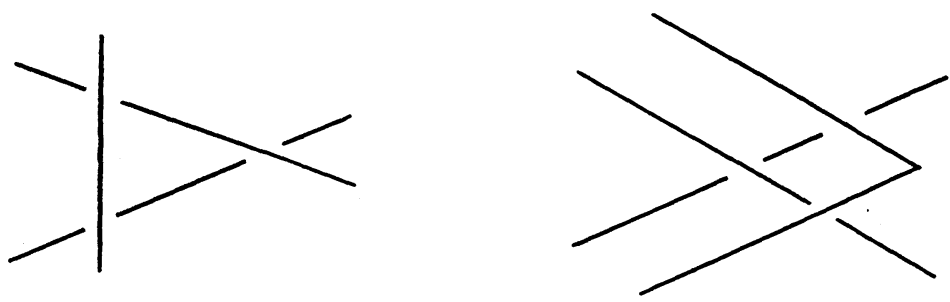

FIGURE 5

The above diagram transformations determine the following possible transformations between the associated words.

( I ) $A \rightarrow A x^{e} x^{-e}(e= \pm 1)$

(II) $A B \rightarrow A x^{e} y^{e} B x^{-e} y^{e}$

$A B \rightarrow A x^{e} y^{e} B y^{-e} x^{-e} \quad(e= \pm 1)$

(III) $A x_{1} y_{1} B x_{1}^{-1} z_{1} C y_{1}^{-1} z_{1}^{-1} \longrightarrow A y_{2} x_{2} B z_{2} x_{2}^{-1} C z_{2}^{-1} y_{2}^{-1}$

$A y_{1} x_{1} B x_{1}^{-1} z_{1} C y_{1}^{-1} z_{1}^{-1} \longrightarrow A x_{2} y_{2} B z_{2} x_{2}^{-1} C z_{2}^{-1} y_{2}^{-1}$

$A x_{1} y_{1} B x_{1}^{-1} z_{1} C z_{1}^{-1} y_{1}^{-1} \longrightarrow A y_{2} x_{2} B z_{2} x_{2}^{-1} C y_{2}^{-1} z_{2}^{-1}$

$A y_{1} x_{1} B x_{1}^{-1} z_{1} C z_{1}^{-1} y_{1}^{-1} \longrightarrow A x_{2} y_{2} B z_{2} x_{2}^{-1} C y_{2}^{-1} z_{2}^{-1}$.

The words in (III) may occur with the order of the pairs $x_{1} y_{1}\left(y_{1} x_{1}\right)$, $x_{1}^{-1} z_{1}, y_{1}^{-1} z_{1}^{-1}\left(z_{1}^{-1} y_{1}^{-1}\right)$ being permuted provided that the analogous rearrangement is made for the pairs $y_{2} x_{2}\left(x_{2} y_{2}\right), z_{2} x_{2}^{-1}, z_{2}^{-1} y_{2}^{-1}\left(y_{2}^{-1} z_{2}^{-1}\right)$.

We now list three additional types of transformations of words.

(0) Any transformation between two equivalent words.

(IV) A transformation which results from a reversal of the orientation of the knot,

$$
\text { i.e., } x_{1}^{e_{1}} \cdots x_{2 n}^{e_{2 n}} \longrightarrow x_{2 n}^{e_{2 n}} \cdots x_{1}^{e_{1}} \text {. }
$$


(V) A transformation which results from a reflection through the $x y$-plane,

$$
\text { i.e., } x_{1}^{e_{1}} \cdots x_{2 n}^{e_{2 n}} \longrightarrow x_{1}^{-e_{1}} \cdots x_{2 n}^{-e_{2 n}} \text {. }
$$

DEFINITION 4.1. A sequence $W_{1}, W_{2}, \cdots, W_{n}$ of words of knots is called a fundamental sequence of words between $U$ and $V$ iff $W_{1}=$ $U, W_{n}=V$ and $W_{i}$ is obtained from $W_{i-1}(i=2, \cdots, n)$ by a transformation of one of the types (0)-(V).

DEFINITION 4.2. A finite sequence of words and characteristics of double points of knots is said to be fundamental if each word and its successor are related by a transformation of one of the types (0)(III) and under this transformation corresponding double points have the same characteristic.

THEOREM 4.3. Let $K, L$ be two prime knots with words $U, V$ respectively. Then $K$ and $L$ belong to the same knot type iff there is a fundamental sequence of words between $U$ and $V$.

Proof. (Necessity) Suppose $K$ and $L$ are prime knots which belong to the same knot type. We regard $K, L$ as oriented knots with the orientations being determined by $U, V$ respectively. Let $\sigma$ denote reversal of knot orientation and let $\rho(x, y, z)=(x, y,-z)$. Then the oriented knot $K$ must belong to the same oriented knot type as one of the oriented knots $L, \rho L, \sigma L, \sigma \rho L$. Hence the necessity of the theorem follows from [1].

(Sufficiency) Let $W_{1}, W_{2}, \cdots, W_{n}$ be a fundamental sequence of words between $U$ and $V$. Let $K_{2}, \cdots, K_{n-1}$ be a sequence of knots with words $W_{2}, \cdots, W_{n-1}$ respectively. If $W_{2}$ is obtained from $W_{1}$ by a type (0) transformation it is easy to show that $W_{1}$ and $W_{2}$ have equivalent prime factorizations. Hence $K$ is equivalent to $K_{2}$ by Theorem 3.5. If $W_{2}$ is not obtained from $W_{1}$ by a type (0) transformation, then it is evident that there exist equivalent knots $L_{1}, L_{2}$ with words $W_{1}, W_{2}$ respectively. By Theorem $3.5 K$ is equivalent to $L_{1}$ and $L_{2}$ is equivalent to $K_{2}$. Thus $K$ is equivalent to $K_{2}$. Using an inductive argument we obtain the desired result that $K$ and $L$ belong to the same knot type.

By using an argument similar to that used above and evoking Theorem 3.4 instead of Theorem 3.5 we obtain

THEOREM 4.4. Two oriented knots $K$ and $L$ belong to the same oriented knot type iff there is a fundamental sequence relating a 
word and the characteristics of the double points of $K$ to a word and the characteristics of the double points of $L$.

Remarks 4.5. L. B. Treybig has conjectured that if $U$ and $V$ are words of length $\leqq m$ which may be connected by a fundamental sequence of words, then there is an integer $N(m)$ and a fundamental sequence of words between $U$ and $V$ such that each word in the sequence has length $\leqq N(m)$. In [13] Treybig obtains some partial results in this direction. The proof of such a conjecture together with Theorem 4.4 would provide an algorithm for determining oriented knot types.

5. The group of a prime word. By Corollary 3.3 there are precisely two sets of characteristics associated with a prime word, one set being the negatives of the other. Given a prime word, a set of characteristics associated with its alphabet may be obtained by simply constructing any diagram associated with the word.

Let $W=b_{1}^{e_{1}} b_{2}^{e_{2}} \cdots b_{2 n}^{e_{2 n}}$ be a prime word. A segment (consider $W$ to be cyclically ordered) of the form $b_{i} \cdots b_{\imath+k}$ where $e_{\imath-1}=e_{i+k+1}=-1$ is called an over segment, and a segment of the form $b_{j}^{-1} \cdots b_{j+m}^{-1}$ where $e_{j-1}=e_{j+m+1}=1$ is called and under segment. The group of $W$ is presented as follows: The alphabet of $W$ is the set of generators. For each over segment $b_{i} \cdots b_{i+k}$ we associate the relation $b_{\imath}=\cdots=b_{i+k}$. For each under segment $b_{j}^{-1} \cdots b_{j+m}^{-1}$ we associate the relation $b_{j-1} b_{j}^{\varepsilon(b j)} \cdots b_{j+m}^{\varepsilon(b j+m)} b_{j+m+1}^{-1} b_{j+m}^{-\varepsilon(b j+m)} \cdots b_{j}^{-\varepsilon(b j)}=1$. The above generators and relations present a group which we shall call the group of $W$.

THEOREM 5.1. A group is a knot group iff it has a presentation which presents the group of a prime word.

Proof. To prove the theorem it suffices to show that every oriented knot type has a representative diagram which possesses a prime word, and that the group of a prime word $W$ is the group of any knot whose diagram yields $W$.

Let $K$ be an oriented knot with word $W$, and let $W_{1}, \cdots, W_{m}$ denote the prime factors of $W$. Then $K$ can be represented as a composition of oriented knots $K_{1}, \cdots, K_{m}$ possessing prime words $W_{1}$, $\cdots, W_{m}$ respectively. If $K$ is a prime knot it follows that only one of $K_{1}, \cdots, K_{m}$ belongs to a nontrivial knot type. Hence it can be assumed that every prime knot possesses a prime word. Thus given an oriented knot $K$, we may assume that $K$ is a composition of oriented prime knots $K_{1}, \cdots, K_{m}$ with prime words $W_{1}, \cdots, W_{m}$ respectively, and that the word of $K$ is $W_{1} W_{2} \cdots W_{m}$. An isotopic deformation 
of $R^{3}$ can be chosen so as to deform an arc in $K_{1}$ so that it passes under $K_{2}, \cdots, K_{m}$ in such a way that $K$ is transformed into a new knot $K^{\prime}$ which possesses a prime word.

Now suppose $K$ is an oriented knot with prime word $W$. We may assume that the characteristics associated with $W$ are the characteristics of $K$. (For otherwise, the characteristics would be associated with the diagram of a knot $\alpha \rho K$ where $\alpha$ is a rotation and $\rho(x, y$, $z)=(x, y,-z)$. Since $K$ and $\alpha \rho K$ are equivalent, it would suffice to show that the presentation for the group of $W$ presents the group of $\alpha \rho K$.) By using the Tietze operations we can eliminate all the generators in the presentation except for the first letter in each over segment. It is then easy to check that the resulting presentation is precisely the over presentation of the group of $K$ given by Fox and Torres (p. 212, [4]).

\section{REFERENCES}

1. J. W. Alexander and G. B. Briggs, On types of knotted curves, Ann. of Math., 28 (1927), 562-586.

2. R. H. Fox, On the complementary domains of a certain pair of inequivalent knots, Ned. Akad. Wetensch. Indag. Math., 14 (1952), 37-40.

3. - A quick trip through knot theory, Topology of 3-Manifolds, Prentice-Hall, Englewood Cliffs, N. J., (1962), 120-167.

4. R. H. Fox and G. Torres, Dual presentations of the group of a knot, Ann. of Math., 59 (1954), 211-218.

5. W. Graeub, Die semilinearen Abbildungen, S.-B. Heidelberger Akad. Wiss. Math. Nat. Kl., (1950), 205-272.

6. M. L. Marx, The Gauss realizability problem, Proc. Amer. Math. Soc., 22 (1969), 610-613.

7. E. E. Moise, Affine structures in 3-manifolds VIII, invariance of the knot types; local tame imbedding, Ann. of Math., 59 (1954), 159-170.

8. D. E. Penney, Establishing isomorphism between tame prime knots in $E^{3}$, Pacific J. Math., 40 (1972), 675-680.

9. K. Reidemeister, Knotentheorie, Chelsea, Now York, 1948.

10. L. B. Treybig, A characterization of the double point structure of the projection of a polygonal knot in regular position, Trans. Amer. Math. Soc., 130 (1968), 223-247. 11. - Prime mappings, Trans. Amer. Math. Soc., 130 (1968), 248-253.

12. - An approach to the polygonal knot problem using projections and isotopies, Trans. Amer. Math. Soc., 158 (1971), 409-421.

13. - Concerning a bound problem in knot theory, Trans. Amer. Math. Soc., 158 (1971), 423-436.

Received October 10, 1972. This paper represents a portion of the author's $\mathrm{Ph}$. D. dissertation, written at Tulane University under the direction of Professor L. B. Treybig, and was supported partly by the National Research Council of Canada (grant A8205).

TUlane UNIVERSITy

AND

The University of Saskatchewan (Saskatoon Campus) 



\section{PACIFIC JOURNAL OF MATHEMATICS}

\section{EDITORS}

RICHARD ARENS (Managing Editor)

University of California

Los Angeles, California 90024

R. A. BeAumont

University of Washington

Seattle, Washington 98105
J. DugundJI*

Department of Mathematics

University of Southern California

Los Angeles, California 90007

D. Gilbarg and J. Milgram

Stanford University

Stanford, California 94305

\section{ASSOCIATE EDITORS}

E. F. BECKENBACH

B. H. NEUMANN

F. WOLF

K. YoSHIDA

\section{SUPPORTING INSTITUTIONS}

UNIVERSITY OF BRITISH COLUMBIA
CALIFORNIA INSTITUTE OF TECHNOLOGY
UNIVERSITY OF CALIFORNIA
MONTANA STATE UNIVERSITY
UNIVERSITY OF NEVADA
NEW MEXICO STATE UNIVERSITY
OREGON STATE UNIVERSITY
UNIVERSITY OF OREGON
OSAKA UNIVERSITY

UNIVERSITY OF BRITISH COLUMBIA CALIFORNIA INSTITUTE OF TECHNOLOGY UNIVERSITY OF CALIFORNIA MONTANA STATE UNIVERSITY NEW MEXICO STATE UNIVERSITY UNIVERSITY OF OREGON OSAKA UNIVERSITY
UNIVERSITY OF SOUTHERN CALIFORNIA STANFORD UNIVERSITY UNIVERSITY OF TOKYO UNIVERSITY OF UTAH WASHINGTON STATE UNIVERSITY UNIVERSITY OF WASHINGTON AMERICAN MATHEMATICAL SOCIETY NAVAL WEAPONS CENTER

* C. R. DePrima California Institute of Technology, Pasadena, CA 91109, will replace J. Dugundji until August 1974. 


\section{Pacific Journal of Mathematics}

\section{Vol. 51, No. $1 \quad$ November, 1974}

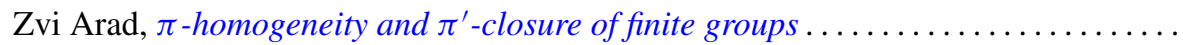

Ivan Baggs, A connected Hausdorff space which is not contained in a maximal

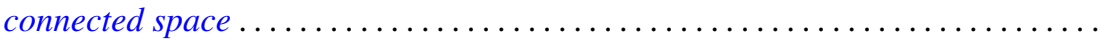

Eric Bedford, The Dirichlet problem for some overdetermined systems on the unit ball in $C^{n}$

R. H. Bing, Woodrow Wilson Bledsoe and R. Daniel Mauldin, Sets generated by

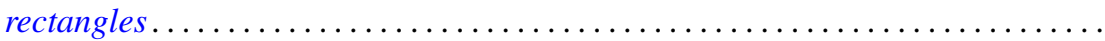

Carlo Cecchini and Alessandro Figà-Talamanca, Projections of uniqueness for

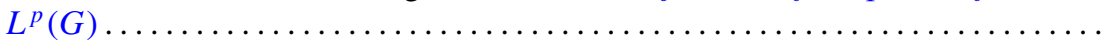

Gokulananda Das and Ram N. Mohapatra, The non absolute Nörlund summability of Fourier series .

Frank Rimi DeMeyer, On separable polynomials over a commutative ring ........ Richard Detmer, Sets which are tame in arcs in $E^{3} \ldots \ldots \ldots \ldots \ldots \ldots \ldots \ldots$

William Erb Dietrich, Ideals in convolution algebras on Abelian groups ..........

Bryce L. Elkins, A Galois theory for linear topological rings .................

William Alan Feldman, A characterization of the topology of compact convergence on $C(X)$.

Hillel Halkin Gershenson, A problem in compact Lie groups and framed cobordism

Samuel R. Gordon, Associators in simple algebras.

Marvin J. Greenberg, Strictly local solutions of Diophantine equations

Jon Craig Helton, Product integrals and inverses in normed rings . . . . . . . . . . . .

Domingo Antonio Herrero, Inner functions under uniform topology . . .

Jerry Alan Johnson, Lipschitz spaces .

Marvin Stanford Keener, Oscillatory solutions and multi-point boundary value

functions for certain nth-order linear ordinary differential equations.

John Cronan Kieffer, A simple proof of the Moy-Perez generalization of the

Shannon-McMillan theorem .......................

Joong Ho Kim, Power invariant rings

Gangaram S. Ladde and V. Lakshmikantham, On flow-invariant sets .

Roger T. Lewis, Oscillation and nonoscillation criteria for some self-adjoint even

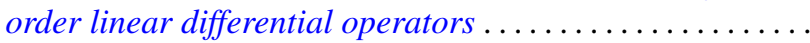

Jürg Thomas Marti, On the existence of support points of solid convex sets ..

John Rowlay Martin, Determining knot types from diagrams of knots . .

James Jerome Metzger, Local ideals in a topological algebra of entire functions

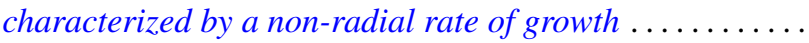

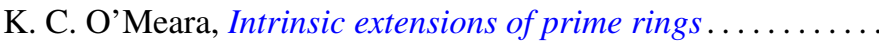

Stanley Poreda, A note on the continuity of best polynomial approximations ..

Robert John Sacker, Asymptotic approach to periodic orbits and local prolongations

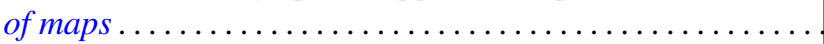

Eric Peter Smith, The Garabedian function of an arbitrary compact set . .

Arne Stray, Pointwise bounded approximation by functions satisfying a side condition

John St. Clair Werth, Jr., Maximal pure subgroups of torsion complete abelian

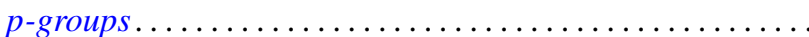

